\title{
Package Identifier
}

National Cancer Institute

\section{Source}

National Cancer Institute. Package Identifier. NCI Thesaurus. Code C93402.

The unique identification of a package in a specific context. 